\title{
Medicina não-convencional: prevalência em pacientes oncológicos*
}

\author{
Non-conventional medicine: prevalence in oncologic patients
}

Mardia Carlos Elias e Elaine Alves²

\section{Resumo}

Este estudo descreve a prevalência de medicina não-convencional em pacientes oncológicos a partir da entrevista de 105 pacientes atendidos no Ambulatório de Q uimioterapia de Adultos do H ospital Universitário de Brasília (H UB) no período de dezembro de 2000 a junho de 2001. Os achados demonstraram que $63,81 \%$ dos pacientes fizeram uso de medicina nãoconvencional. O s usuários foram, em sua maioria, mulheres $(p=0,417)$, jovens, brancas ( $p=0,856)$, com câncer de mama, residentes no $D$ istrito Federal $(p=0,317)$, com boas condições de moradia e com ensino fundamental incompleto $(p=0,347)$. A fitoterapia foi a terapia mais utilizada. A razão mais freqüentemente mencionada para o uso das terapias não-convencionais foi a indicação de terceiros, com o objetivo de curar a doença. A medicina não-convencional foi analisada com ênfase nos fatores que influenciam sua prevalência na oncologia, sua relação com a enfermagem e com a ética. $D$ iscorreu-se a respeito dos riscos, benefícios e precauções em relação às terapias não-convencionais.

Palavras-chave: neoplasias; pacientes; medicina alternativa; prevalência.

\section{Abstract}

This research is about the prevalence of non-conventional medicine in 105 cancer outpatients from the Service of Clinical Oncology at University of Braślia H ospital surveyed by means of an interview between December 2000 and June 2001. Of the 105 participants, $63,81 \%$ used non-conventional therapies. $N$ on-conventional medicine users were mostly white $(p=0,856)$ young women $(p=0,417)$, with breast cancer, who had not completed the elementary school, living in Distrito Federal $(p=0,317)$ with satisfactory housing conditions. U se was greatest for herbs. M ost patients used non-conventional therapies to cure cancer. $\mathrm{N}$ on-conventional medicine is discussed in this paper,focusing its prevalence in 0 ncology and its relation to nursing and ethics. It was addressed the precautions, risks and benefits of non-conventional medicine.

Key words neoplasms; patients, alternative medicine; prevalence.

*Resumo da dissertação de mestrado apresentada no Curso de Pós-Graduação em Ciências da Saúde da Faculdade de Ciências da Saúde (FS), U niversidade de Braślia (UnB).

${ }^{1}$ Enfermeira. M estre em Ciências da Saúde pelo Curso de Pós-Graduação em Ciências da Saúde da FS - UnB.

${ }^{2}$ Professora Adjunta da Área de M edicina da Criança e do Adolescente da Faculdade de M edicina - UnB. Chefe do Serviço de 0 ncologia Pediátrica do Centro de Clínicas de Cirurgia Pediátrica do H ospital Universitário da UnB. D outora em M edicina pela Escola Paulista de M edicina - UN IFESP. Professora O rientadora. Enviar correspondência para E.A.

E-mail: daephub@unb.br

Recebido em março de 2002. 


\section{INTRO DUÇÃO}

As terapias não-convencionais: sua relação com a oncologia

D esde a última década tem sido observada uma atenção crescente quanto ao uso da medicina não-convencional, utilizada em muitos países desenvolvidos. As terapias nãoconvencionais são descritas como um conjunto de práticas de prevenção, diagnóstico e tratamento à parte do modelo médico dominante. Práticas que não são, comumente, ensinadas nas escolas médicas ou executadas nos hospitais e cuja eficácia é questionada por não haver estudos rigorosos para a análise de resultados e pela falta de uma base racional à luz dos conhecimentos científicos atuais. ${ }^{1}$

Fletcher $^{2}$ descreve a falta de uma uniformidade na cura do câncer e a necessidade de reduzir a ansiedade e de retomar o controle da saúde como as possíveis razões para o paciente oncológico buscar a medicina não-convencional. As fases mais críticas seriam: o momento do diagnóstico, o diagnóstico de metástases, a recorrência e durante estágios avançados da doença. N essas ocasiões o paciente pode se tornar mais vulnerável e procurar tratamentos nãoconvencionais.

Entre os profissionais de saúde que trabalham com pacientes oncológicos, tem surgido uma preocupação quanto ao uso de métodos terapêuticos alternativos ou complementares e 0 risco do paciente abandonar a medicina tradicional no tratamento do câncer e optar pela medicina não-convencional, prejudicando as chances de sucesso do tratamento da doença, principalmente em sua fase inicial. Também são levantadas questões a respeito de interações indesejáveis entre o tratamento padrão do câncer e terapêuticas não-

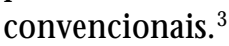

0 tratamento do câncer foi um processo lento no seu desenvolvimento. O s médicos e cirurgiões antigos reconheciam que o câncer, em geral, recidivava após ter sido removido cirurgicamente. Esta constatação implicou em considerar o câncer uma doença incurável, conceito que permaneceu por muitos anos, até mesmo no século $X X$.

Atualmente, o cuidado do paciente oncológico continua fragmentado e os pacientes se queixam do diagnóstico tardio, da má comunicação com os médicos e outros profissionais de saúde e da falta de informação e suporte adequados. ${ }^{4} \mathrm{Com}$ a doença, particularmente o câncer, o indivíduo sente-se intimidado com a possibilidade do fim de sua existência. A ansiedade gerada determina comportamentos variados e leva à busca de outras formas de tratamento. Assim, as práticas não-convencionais de tratamento dão ao paciente oncológico mais uma opção e complementação ao tratamento oficial a ele sugerido. ${ }^{5}$ U ma das razões para 0 interesse atual em medicina alternativa é a insatisfação com a natureza tecnológica e impessoal da medicina moderna; os pacientes reclamam de um relacionamento insensível, limitado e apressado com os oncologistas, e se sentem ignorados e sem auxílio. Em relação aos profissionais de medicina alternativa, os pacientes os consideram mais atenciosos ao tratarem a pessoa como um todo. ${ }^{6}$

Sabe-se que a maior parte dos pacientes faz uso dessas práticas sem que os médicos e enfermeiros sejam informados. As razões são diversas e incluem a percepção da falta de interesse do médico em ouvir a respeito do assunto, a antecipação de uma reação negativa e a crença de que os médicos não possuem conhecimento e treinamento adequados a respeito de medicina alternativa. ${ }^{7}$

É importante entender o que os pacientes procuram nos tratamentos alternativos e como os escolhem. Isto requer sensibilidade quanto à diversidade cultural, social e étnica dos pacientes. $^{8}$

\section{Objetivos do estudo}

Em nosso meio é desconhecido o número de pacientes oncológicos que utilizam terapias não-convencionais. 0 uso dessas terapias pode ser responsável, em certas condições, pelo agravamento do curso da doença, assim como dos efeitos colaterais do tratamento oferecido a esses pacientes. Tais fatos nos levaram a analisar a prevalência do uso de medicina nãoconvencional pelos pacientes portadores de câncer atendidos no H ospital U niversitário da Universidade de Braślia, uma instituição pública de saúde prestadora de assistência oncológica no Distrito Federal. 
O s objetivos específicos foram:

- caracterizar os aspectos sócio-demográficos e clínico-epidemiológicos dos pacientes oncológicos ambulatoriais usuários de medicina não-convencional;

- estimar a prevalência de terapias nãoconvencionais nesses pacientes;

- conhecer os tipos de terapia nãoconvencional utilizados;

- identificar as razões para o uso de terapia não-convencional;

- verificar o tempo de uso da terapia nãoconvencional;

- pesquisar se o paciente declarou à equipe médica a utilização de terapia nãoconvencional;

- verificar o desejo do paciente de receber informação sobre terapia não-convencional; - conhecer o grau de orientação oferecida aos pacientes sobre a terapia não-convencional pelos profissionais de saúde.

\section{MÉTO DOS}

\section{CAsuística}

Foi obtida a partir do censo dos pacientes adultos atendidos no Ambulatório de Q uimioterapia do Serviço de O ncologia Clínica do Hospital Universitário da UnB (HUB), no período de dezembro de 2000 a junho de 2001. D e um total de 182 pacientes, foram entrevistados 105. N ão comparecimento ao hospital, óbito, mudança de endereço e telefone, e viagens foram as razões que impossibilitaram a entrevista de 77 pacientes. $\mathrm{N}$ ão houve recusa em participar da entrevista e responder ao questionário.

Os critérios de inclusão foram: ser portador de neoplasia maligna; idade igual ou superior a 18 anos; doença ativa, em remissão ou recaída; pacientes de ambos os sexos; tomar conhecimento ou assinar o "Termo de Consentimento Livre e Esclarecido". Foram excluídos os pacientes que não atendiam a esses critérios e aqueles portadores de doença mental ou câncer em fase terminal.

\section{PROCEDIMENTO}

A coleta dos dados foi pelo método qualitativo-quantitativo, com a finalidade de levantar as características dos participantes da pesquisa. As variáveis pesquisadas foram: identificação (nome, data de nascimento, sexo, estado civil, registro de prontuário médico); procedência e naturalidade; grau de escolaridade; profissão e renda familiar; religião; etnia; condições de moradia; história médica (diagnóstico, data do diagnóstico, expectativa de sobrevida, tempo em tratamento médico, tempo sem tratamento médico, forma de tratamento em uso ou utilizado, região do corpo irradiada, tipo de cirurgia realizada, outras doenças existentes); efeitos colaterais (efeitos adversos do tratamento convencional que foram, segundo informações do paciente, mais freqüentes); prática de medicina não-convencional (época do início do tratamento não-convencional, se o paciente informou ao seu médico quanto ao uso da medicina não-convencional, se o paciente recebeu orientação da equipe médica a respeito e, ainda, se 0 mesmo gostaria de receber informações quanto à medicina nãoconvencional).

Para a coleta e registro dos dados foi elaborado um questionário composto de indagações relacionadas com as variáveis acima descritas e elaborado no Page $M$ aker, versão 6.0. 0 questionário foi aplicado exclusivamente pela pesquisadora MCE, pessoalmente ou por telefone. Dos 105 pacientes, 62 foram entrevistados pessoalmente e 43 por via telefônica. No momento da entrevista foi explicado ao paciente o significado de medicina nãoconvencional.

Para a realização desta pesquisa foi solicitada e obtida, previamente, a autorização do Comitê de Ética em Pesquisa da Faculdade de M edicina da Universidade de Braślia.

0 programa de entrada de dados utilizado foi o Fox Pro, versão 6.1. A análise dos dados foi programada no System Analysis Statistical SAS, versão 6.12. A análise estatística das diferenças dos usuários e não usuários de medicina não-convencional foi baseada no Teste Exato de Fisher para as variáveis categóricas e no Teste $t$ para as variáveis contínuas. 0 nível de significância $(\alpha)$ requerido foi 0,05 . 


\section{RESULTADO S}

\section{GERAIS}

\section{Aspectos sócio-demográficos}

Dos entrevistados, 56 eram do sexo feminino $(53,33 \%)$ e 49 do sexo masculino (46,66\%). A média da idade foi de 52 anos. Cerca de $25 \%$ dos pacientes nasceram em $M$ inas Gerais (Tabela 1). A grande maioria residia no Distrito Federal e no Entorno (Tabela 2). O Entorno compreende os municípios de Goiás e Minas Gerais circunvizinhos ao Distrito Federal.

Predominaram os pacientes de raça branca (Tabela 3) e de religião católica (Tabela 4). $M$ ais da metade dos entrevistados eram casados (Tabela 5). Q uanto à profissão (Tabela 6), 31 pacientes exerciam atividades do lar, correspondendo a cerca de $30 \%$ do total.

Tabela 1. Naturalidade dos 105 pacientes entrevistados, atendidos no Ambulatório de Q uimioterapia de Adultos do HUB, de dezembro de 2000 a junho de 2001.

\begin{tabular}{|l|c|}
\hline \multicolumn{1}{|c|}{ Naturalidade } & Número de pacientes (\%) \\
\hline Minas Gerais & $26(24,76)$ \\
\hline Goiás & $14(13,33)$ \\
\hline Bahia & $13(12,38)$ \\
\hline Maranhão & $11(10,47)$ \\
\hline Outros & $41(39,04)$ \\
\hline
\end{tabular}

Tabela 2. Procedência dos 105 pacientes entrevistados, atendidos no Ambulatório de Q uimioterapia de Adultos do HUB, de dezembro de 2000 a junho de 2001.

\begin{tabular}{|l|c|}
\hline \multicolumn{1}{|c|}{ Procedência } & Número de pacientes (\%) \\
\hline Distrito Federal & $78(74,28)$ \\
\hline Entorno & $23(21,9)$ \\
\hline Outros & $04(3,8)$ \\
\hline
\end{tabular}

Em relação à escolaridade (Tabela 7), a mai oria dos entrevistados tinha o ensino fundamental incompleto. Q uanto às condições de moradia, 93 pacientes residiam em casa de alvenaria $(88,57 \%) ; 98$ residências contavam com água encanada (93,33\%), 73 eram ligadas à rede de esgoto $(69,52 \%)$ e 91 eram atendidas pelo serviço público de coleta de lixo $(86,67 \%)$. A renda familiar média foi de quatro salários-mínimos.
Tabela 3. Etnia dos 105 pacientes entrevistados, atendidos no Ambulatório de Q uimioterapia de Adultos do HUB, de dezembro de 2000 a junho de 2001.

\begin{tabular}{|l|c|}
\hline \multicolumn{1}{|c|}{ Etnia } & Número de pacientes (\%) \\
\hline Brancos & $57(54,28)$ \\
\hline Pardos & $41(39,04)$ \\
\hline Negros & $07(6,66)$ \\
\hline
\end{tabular}

Tabela 4. Crença religiosa dos 105 pacientes entrevistados, atendidos no Ambulatório de Q uimioterapia de Adultos do HUB, de dezembro de 2000 a junho de 2001.

\begin{tabular}{|l|c|}
\hline \multicolumn{1}{|c|}{ Religião } & Número de pacientes (\%) \\
\hline Católica & $84(80)$ \\
\hline Protestante & $19(18,09)$ \\
\hline Espírita & $02(1,9)$ \\
\hline
\end{tabular}

Tabela 5. Estado civil dos 105 pacientes entrevistados, atendidos no Ambulatório de $\mathrm{Q}$ uimioterapia de Adultos do HUB, de dezembro de 2000 a junho de 2001.

\begin{tabular}{|l|c|}
\hline \multicolumn{1}{|c|}{ Estado Civil } & Número de pacientes (\%) \\
\hline Casados & $59(56,19)$ \\
\hline Solteiros & $18(17,14)$ \\
\hline Viúvos & $12(11,42)$ \\
\hline Divorciados & $08(7,61)$ \\
\hline Regime de concubinato & $08(7,61)$ \\
\hline
\end{tabular}

Tabela 6. Profissão dos 105 pacientes entrevistados, atendidos no Ambulatório de $\mathrm{Q}$ uimioterapia de Adultos do HUB, de dezembro de 2000 a junho de 2001.

\begin{tabular}{|l|c|}
\hline \multicolumn{1}{|c|}{ Profissão } & Número de pacientes (\%) \\
\hline Atividades do lar & $31(29,52)$ \\
\hline Aposentado(a) & $11(10,47)$ \\
\hline Lavrador & $09(8,57)$ \\
\hline Outras & $54(51,42)$ \\
\hline
\end{tabular}

Tabela 7. Escolaridade dos 105 pacientes entrevistados, atendidos no Ambulatório de Q uimioterapia de Adultos do HUB, de dezembro de 2000 a junho de 2001 . O ensino fundamental corresponde ao antigo ensino de 1.0 grau e o ensino médio ao antigo ensino de 2.0 grau.

\begin{tabular}{|l|c|}
\hline \multicolumn{1}{|c|}{ Escolaridade } & $\begin{array}{c}\text { Número de } \\
\text { pacientes }(\%)\end{array}$ \\
\hline $\begin{array}{l}\text { Ensino Fundamental } \\
\text { incompleto }\end{array}$ & $44(41,9)$ \\
\hline Ensino Médio completo & $15(14,28)$ \\
\hline Analfabetos & $13(12,38)$ \\
\hline Ensino Fundamental completo & $11(10,47)$ \\
\hline Ensino Médio incompleto & $10(9,52)$ \\
\hline Ensino Superior completo & $05(4,76)$ \\
\hline Pós-Graduação & $04(3,8)$ \\
\hline Ensino Superior incompleto & $03(2,85)$ \\
\hline
\end{tabular}


Aspectos dínico-epidemiológicos

As neoplasias mais freqüentes foram: câncer de mama, linfomas e câncer de pulmão (Tabela 8). 0 tempo médio decorrido entre 0 diagnóstico da doença e a realização da entrevista foi de 19 meses. Trinta e oito pacientes estavam em remissão da doença e 25 apresentavam recidiva (Tabela 9); 97 estavam na vigência do tratamento médico convencional e os demais eram apenas acompanhados clinicamente. A duração média do tratamento convencional foi de quatro meses. As modalidades terapêuticas tradicionais utilizadas estão relacionadas na Tabela 10, havendo, com freqüência, associação das mesmas. Dentre as intervenções cirúrgicas, a mais freqüente foi a exérese do tumor ( $32,38 \%$ do total); a região mais irradiada foi o tórax $(13,33 \%)$.

Tabela 8. Diagnósticos dos 105 pacientes entrevistados, atendidos no Ambulatório de Q uimioterapia de Adultos do HUB, de dezembro de 2000 a junho de 2001.

\begin{tabular}{|l|c|}
\hline \multicolumn{1}{|c|}{ Diagnósticos } & $\begin{array}{c}\text { Número de } \\
\text { pacientes (\%) }\end{array}$ \\
\hline Câncer de mama & $24(22,85)$ \\
\hline Linfomas & $20(19,04)$ \\
\hline Câncer de pulmão & $11(10,48)$ \\
\hline Outros & $50(47,61)$ \\
\hline
\end{tabular}

Tabela 9. Evolução da doença nos 105 pacientes entrevistados, Atendidos no Ambulatório de Q uimioterapia de Adultos do HUB, de dezembro de 2000 a junho de 2001.

\begin{tabular}{|l|c|}
\hline \multicolumn{1}{|c|}{ Evolução da doença } & $\begin{array}{c}\text { Número de } \\
\text { pacientes }(\%)\end{array}$ \\
\hline Fase inicial do tratamento & $42(40)$ \\
\hline Doença em remissão & $38(36,19)$ \\
\hline Doença em recidiva & $25(23,8)$ \\
\hline
\end{tabular}

Os pacientes relataram os efeitos colaterais usuais provocados pelo tratamento convencional (Tabela 11) e 35 (33,33\%) fizeram referência a outras doenças concomitantes. D estas, a hipertensão arterial sistêmica foi a mais freqüente, correspondendo a $60 \%$ das citadas.

Dos 105 pacientes entrevistados, 67 $(63,81 \%)$ afirmaram usar ou terem usado medicina não-convencional (Figura 1). N oventa e cinco pacientes $(90,47 \%)$ referiram não ter recebido qualquer orientação ou esclarecimento, por parte da equipe médica, quanto à medicina não-convencional, mas 82 $(78,09 \%)$ manifestaram o desejo de receber esclarecimentos sobre 0 assunto.

Tabela 10. Modalidades terapêuticas utilizadas nos 105 pacientes entrevistados, atendidos no Ambulatório de Q uimioterapia de Adultos do HUB, de dezembro de 2000 a junho de 2001.

\begin{tabular}{|l|c|}
\hline \multicolumn{1}{|c|}{ Modalidades terapêuticas } & $\begin{array}{c}\text { Número de } \\
\text { pacientes (\%) }\end{array}$ \\
\hline Quimioterapia & $94(89,52)$ \\
\hline Cirurgia & $62(59,04)$ \\
\hline Radioterapia & $34(32,38)$ \\
\hline
\end{tabular}

Tabela 11. Efeitos colaterais do tratamento convencional nos 105 Pacientes entrevistados, atendidos no Ambulatório de $\mathrm{Q}$ uimioterapia de Adultos do HUB, de dezembro de 2000 a junho de 2001.

\begin{tabular}{|l|c|}
\hline $\begin{array}{c}\text { Efeitos colaterais do } \\
\text { tratamento convencional }\end{array}$ & $\%$ \\
\hline Alopécia & 72,38 \\
\hline Inapetência & 69,52 \\
\hline Náuseas & 68,57 \\
\hline Fadiga & 66,67 \\
\hline Vômitos & 58,10 \\
\hline
\end{tabular}

\section{USUÁRIOS DE MEDICINA NÃO-CONVENCIONAL}

Aspectos sócio-demográficos

Dos usuários, 38 eram do sexo feminino $(56,71 \%)$ e 29 do sexo masculino $(43,29 \%)$. A média de idade foi de 50 anos; considerando-se separadamente mulheres e homens, a idade média foi: 48 anos para mulheres e 52 anos para homens. Em sua maioria eram brancos $(55,22 \%)$, casados $(55,22 \%)$, católicos $(82,09 \%)$, com escolaridade correspondente ao ensino fundamental incompleto $(38,81 \%)$. Todos eram residentes no Distrito Federal e Entorno. A média da renda familiar foi de seis salários mínimos. 
Figura 1. Uso de medicina não convencional pelos pacientes atendidos no Ambulatório de Quimioterapia de Adultos do HUB, de dezembro de 2000 a junho de 2001.

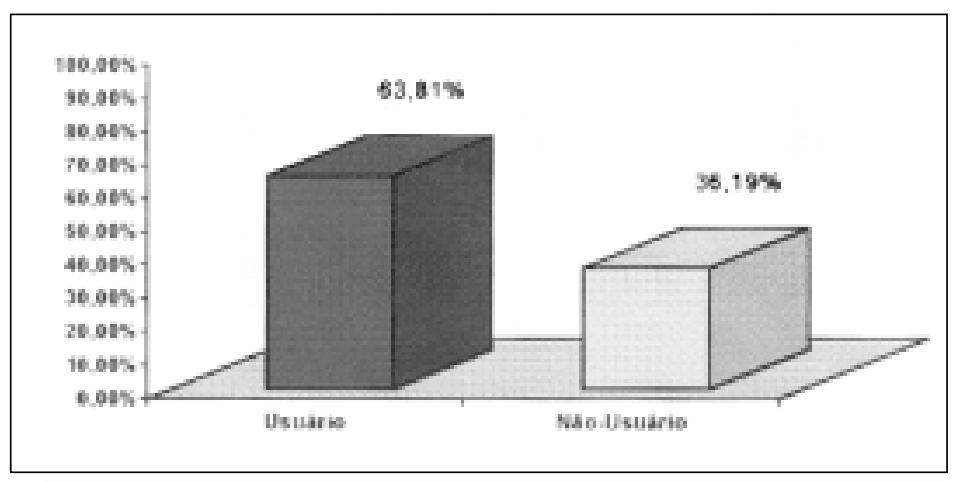

Aspectos clínico-epidemiológicos

Estão relacionados na Tabela 12. D os 67 usuários, $59(88,06 \%)$ haviam sido tratados com quimioterapia, $41(61,19 \%)$ submetidos a intervenções cirúrgicas, 27 (40,3\%) fizeram uso de radioterapia. A duração média do tratamento convencional foi quatro meses. A média de tempo em uso de medicina nãoconvencional foi três meses.

Tabela 12. Aspectos clínico-epidemiológicos dos 67 usuários de medicina não-convencional.

\begin{tabular}{|l|c|c|}
\hline \multicolumn{1}{|c|}{ Aspectos Clinicoepidemiológicos } & $\begin{array}{c}\text { Número de } \\
\text { pacientes }\end{array}$ & $\%$ \\
\hline Câncer de mama & 20 & 29,85 \\
\hline Em tratamento convencional & 60 & 89,55 \\
\hline Doença em recidiva & 19 & 28,35 \\
\hline Doença em remissão & 21 & 31,34 \\
\hline Portador de outras doenças & 22 & 32,83 \\
\hline $\begin{array}{l}\text { Informaram o uso de medicina não- } \\
\text { convencional }\end{array}$ & 30 & 44,77 \\
\hline $\begin{array}{l}\text { Desejo de orientação a respeito de } \\
\text { terapias alternativas }\end{array}$ & 60 & 89,55 \\
\hline
\end{tabular}

A fitoterapia foi a modalidade mais usada de tratamento não-convencional e, quando considerada isoladamente, representou $71,64 \%$ do total. Em conjunto com outras terapias, correspondeu a $92,53 \%$. A homeopatia e os chás tiveram a mesma freqüência de uso $(5,97 \%)$. A associação de diferentes modalidades de tratamento não convencional foi observada em $20,89 \%$ dos usuários (Tabela 13).

A principal razão para o uso das terapias não-convencionais, correspondendo a $71,64 \%$ dos casos, foi a indicação de terceiros (Tabela 14).

Ao se comparar os usuários de medicina não-convencional com os não-usuários, foi observado que estesúltimostinham umaidade média de 56 anos e renda familiar de 2,5 salários mínimos. Não houve diferenças significantes entre os dois grupos, exceto que dentre os usuários $40,30 \%$ haviam sido submetidos à radioterapia, enquanto que entre os não usuários apenas $18,42 \%(p=0,029)$. 0 desejo de orientação em relação à medicina não-convencional foi manifestado por $89,55 \%$ dos usuários e por apenas 10,44\% dos não usuários $(p=0,004)$.

Tabela 13. Terapias não-convencionais usadas por 67 pacientes atendidos no Ambulatório de Q uimioterapia de Adultos do HUB, de dezembro de 2000 a junho de 2001.

\begin{tabular}{|l|c|r|}
\hline \multicolumn{1}{|c|}{ Terapias não-convencionais } & $\begin{array}{c}\text { Número de } \\
\text { pacientes }\end{array}$ & \multicolumn{1}{c|}{$\%$} \\
\hline Ervas & 48 & 71,64 \\
\hline Chás & 2 & 2,99 \\
\hline Ervas+Chás & 2 & 2,99 \\
\hline Ervas+Cirurgia espiritual & 2 & 2,99 \\
\hline Ervas+Benzeduras & 2 & 2,99 \\
\hline Ervas+Homeopatia & 2 & 2,99 \\
\hline Ervas+Medicações & 1 & 1,49 \\
\hline Ervas+Medicações+Homeopatia & 1 & 1,49 \\
\hline Ervas+Medicações+Acupuntura & 1 & 1,49 \\
\hline Ervas+Vitaminas & 1 & 1,49 \\
\hline Ervas+Dietas & 1 & 1,49 \\
\hline Ervas+Rezas & 1 & 1,49 \\
\hline Dietas & 1 & 1,49 \\
\hline Homeopatia & 1 & 1,49 \\
\hline Orações & 1 & 1,49 \\
\hline Total & 67 & 100,00 \\
\hline
\end{tabular}

Tabela 14. Razões para o uso de terapias nãoconvencionais em 67 pacientes atendidos no Ambulatório de Quimioterapia de Adultos do HUB, de dezembro de 2000 a junho de 2001.

\begin{tabular}{|l|c|c|}
\hline \multicolumn{1}{|c|}{ Motivo } & $\begin{array}{c}\text { Número de } \\
\text { pacientes }\end{array}$ & $\%$ \\
\hline $\begin{array}{l}\text { Indicação de terceiros (amigos, } \\
\text { familiares, vizinhos) }\end{array}$ & 48 & 71,64 \\
\hline Mídia (programas de televisão) & 6 & 8,96 \\
\hline Costume familiar & 6 & 8,96 \\
\hline Fé & 3 & 4,48 \\
\hline Medo do tratamento convencional & 2 & 2,99 \\
\hline Cultura & 1 & 1,49 \\
\hline Medo de morrer & 1 & 1,49 \\
\hline
\end{tabular}

\section{DISCUSSÃO}

A medicina não-convencional é constituída por práticas de prevenção, diagnóstico e tratamento que não são ensinadas nas escolas médicas oficiais. Seus defensores a legitimam por proporcionar resultados em uma variedade de afecções, principalmente as crônicas, a partir da concepção de um cuidado que integra a 
totalidadedo ser, quenão existiria na medicina ortodoxa.

As práticas não-convencionais têm atraído um interesse expressivo, tanto da população quanto de profissionais variados. M as, ao lado desse interesse, observa-se uma apreensão quanto às razões para o seu uso, a carência de comprovação científica, a falta de regulamentação, a propaganda exacerbada nos meios de comunicação, os efeitos adversos possíveis e a qualificação dos profissionais. Essa apreensão, ao contrário do que se poderia imaginar, não se origina apenas entre os oponentes da medicina não-convencional. Ela também é percebida entre profissionais que a exercem e desejam uma aplicação bem orientada da mesma.

0 uso da medicina não-convencional éum fenômeno universal. M uitas modalidades terapêuticas empregadas certamente contribuem para o bem-estar do indivíduo, ainda que não se compreenda completamente seus mecanismos de ação. A sua prevalência é estimada em $25 \%$ no Reino Unido, $50 \%$ na Alemanha, França e Austrália e entre 42\% e $69 \%$ na população dos Estados U nidos. ${ }^{9}$

A variação da prevalência do uso de medicina não-convencional nas diferentes sociedades possivelmente decorre, também, da dificuldade em se definir medicina nãoconvencional. D a mesma forma, ainda não é bem estabelecida a prevalência dessa medicina entre pacientes oncológicos. Sabe-se, contudo, que um número significante de pacientes oncológicos usa ou considera seriamente a possibilidade de usar tratamentos nãoconvencionais e quando isso ocorre o profissional da área da oncologia pode se defrontar com complexas questões profissionais, éticas e legais.

0 estudo de Cassileth $^{10}$ realizado nos Estados U nidos da América, revela que 83,3\% dos pacientes oncológicos usam terapias nãoconvencionais. Seus usuários a utilizam mais de forma a complementar o tratamento convencional do que para substituí-lo e, em geral, são jovens, do sexo feminino, com grau de instrução e padrão sócio-econômico mais elevados.

$\mathrm{Na}$ presente pesquisa, $63,81 \%$ dos pacientes entrevistados afirmaram usar ou terem usado medicina não-convencional. As principais razões para esse uso foram as sugestões ou indicações recebidas de amigos, vizinhos ou familiares; as informações obtidas por meio da mídia a respeito de determinados tratamentos que poderiam curar o câncer e, ainda, porque acreditavam em medicina nãoconvencional. Foi verificado que, em sua maioria, eram mulheres brancas, jovens, residentes no $D$ istrito Federal e portadoras de câncer de mama. Essas mulheres tinham renda familiar em torno de seis salários mínimos, não haviam concluído o ensino fundamental e exerciam atividades do lar.

$\mathrm{N}$ ossos achados se assemelham parcialmente aos de outras pesquisas. 0 câncer de mama, os linfomas e o câncer de pulmão foram os diagnósticos mais freqüentes; a maior parte dos estudos não relata nenhuma relação entre o uso da medicina não-convencional e o sítio da doença. Os pacientes que analisamos iniciaram 0 uso da medicina nãoconvencional quase imediatamente após 0 diagnóstico da doença e o início do tratamento convencional. D entre os que empregavam terapias não-convencionais, $31,34 \%$ estavam em remissão e $28,36 \%$ apresentavam recidiva. Poucos estudos revelaram a ocasião em que a opção ocorreria, alguns sugeriram que seria quando da progressão ou recidiva da doença. 2,10,11

0 receio da escolha da medicina nãoconvencional em detrimento do tratamento oficial não parece ser, como temem alguns, algo de grande relevância, ainda que não se deva de todo desprezá-lo. São inquestionáveis os avanços da medicina moderna em proporcionar a cura e controle das doenças. Também são inegáveis as distorções e contradições que essa mesma medicina tem gerado. Iatrogenias da medicina ortodoxa, falta de confiança na medicina oficial, alto custo do tratamento, incapacidade da medicina oficial em solucionar as doenças crônico-degenerativas, falta de acesso à medicina oficial e o desejo por uma assistência holística são motivações constantes na busca de terapias nãoconvencionais.

No presente trabalho foi constatado que entre os usuários de medicina nãoconvencional, $89,55 \%$ estavam em tratamento 
convencional. O s demais pacientes estavam em acompanhamento médico periódico, que é padrão para os portadores de câncer. $\mathrm{N}$ enhum paciente relatou ter optado, primeiramente, pela medicina nãoconvencional e depois ter decidido pelo tratamento ortodoxo. Embora reconhecessem que o tratamento oncológico é diferente dos tratamentos de outras doenças, ou seja, implica em um desgaste físico e emocional importante podendo não proporcionar a cura definitiva, não mostraram descrédito para com a medicina oficial, mas a percepção das limitações do tratamento convencional. Isso pode comprovar o que foi sugerido em outras investigações: os pacientes confiam na medicina convencional eusam as terapias nãoconvencionais complementando o tratamento a que estão sendo submetidos. ${ }^{10-12}$ Eles desejam tentar usar tudo o que Ihes for possível para recuperar a saúde. C oncordamos com M oore ${ }^{12}$ quanto à necessidade de se investigar, com maior cuidado, as razões que levam o paciente a adotar a medicina nãoconvencional.

Vários pacientes utilizavam mais de uma modalidade de tratamento não-convencional, sendo que a fitoterapia foi a mais usada, geralmente, por indicação de terceiros. É o método terapêutico alternativo mais comumente citado nos diferentes trabalhos, possivelmente em razão de ser empregado desde os primórdios da medicina e considerado como natural e inofensivo. ${ }^{13} \mathrm{~N}$ ão há, ainda, uma estimativa oficial a respeito do uso da fitoterapia entre os pacientes com câncer em nosso país, mas acreditamos ser largamente utilizada.

$\mathrm{N}$ ão foi objeto da pesquisa indagar a respeito da possível ocorrência de efeitos colaterais provocados pelos tratamentos nãoconvencionais. Entretanto, três pacientes relataram reações adversas com o uso de ervas. Apesar de considerados inofensivos, os fitoterápicos podem mascarar ou mesmo distorcer os efeitos do tratamento convencional, embora também possam aliviar os efeitos colaterais. 0 potencial para a ocorrência de interações entre fitoterápicos e medicamentos é significativo, principalmente nos pacientes com alteração da função renal. O s pacientes em tratamento radioterápico podem apresentar fotossensibilização cutânea e outras reações graves. 0 uso de fitoterápicos deve ser suspenso antes das intervenções cirúrgicas pelos riscos de interações com anestésicos, hipertensão, hipoglicemia, isquemia cardíaca, hemorragia e rejeição de órgãos transplantados.

O s aspectos referentes à regulamentação e segurança dos fitoterápicos são uma preocupação. Essas drogas não são avaliadas quanto à segurança e eficácia, como é feito para as drogas alopáticas. Muitos dos fitoterápicos são comercializados em diferentes tipos de estabelecimentos, principalmente no mercado informal, sem que haja qualquer controle quanto ao saneamento das instalações e equipamentos, pureza, constituição, identificação, conservação e procedência. ${ }^{14-16}$ Assim, 0 uso de fitoterápicos por pacientes em tratamento de câncer deve ser considerado com cautela.

A quase totalidade dos entrevistados não recebeu quaisquer esclarecimentos sobre a medicina não-convencional, embora uma parcela significativa dos que usaram ou estavam usando tratamentos nãoconvencionais tenha relatado esse fato à equipe médica. $\mathrm{N}$ a maioria das pesquisas há o relato de que os pacientes costumam ocultar essa informação, uma vez que antecipam uma reação negativa.

No presente trabalho, quando perguntados se haviam informado ou não que estavam fazendo uso de medicina nãoconvencional, ao responderem positivamente os pacientes desejavam explicar a razão, que era o desejo de conhecer a opinião da equipe médica a respeito das terapias nãoconvencionais que estavam usando. Receberam respostas como, por exemplo, "não há comprovação científica", que se as referidas terapias "bem não fazem tampouco mal podem fazer", "não conheciam a terapia" e que "era perda de tempo e de dinheiro". 0 utras respostas eram de incentivo.

A análise dos dados mostrou que os usuários e não-usuários de medicina nãoconvencional não diferiram com respeito à etnia, estado civil, idade, sexo, religião, escolaridade e moradia. Também não houve diferença em relação ao fato da doença estar 
em remissão ou progressão. Q uanto ao tipo de tratamento convencional usado observouse significância estatística em relação à radioterapia. Acreditamos que isso se relacione ao tipo de câncer que predominou no universo dos entrevistados.

0 grau de orientação recebida e o desejo de esclarecimentos sobre as terapias nãoconvencionais foram significativos. Interessante foi o fato de se perceber desejo de orientação mesmo entre pacientes não usuários de medicina não-convencional.

O s achados da pesquisa evidenciam não apenas a necessidade de se melhorar a comunicação entre o profissional de saúde e o paciente, mas também do aperfeiçoamento do profissional em conhecer a eficácia, segurança, indicações, contra-indicações, riscos e benefícios do uso da medicina nãoconvencional, incluindo-se o potencial de interações com as terapias convencionais.

Diante do grande interesse dos pacientes pela medicina não-convencional, o profissional da saúde deve se informar a respeito da mesma. A falta de comprovação científica não serve como argumento para ignorar 0 assunto. É um direito do paciente ser esclarecido quanto às possibilidades terapêuticas para a sua doença. ${ }^{17} 0$ paciente deve se sentir à vontade para abordar 0 assunto com aqueles que Ihe prestam assistência e por sua vez 0 profissional de saúde deveria discutir o tema de forma neutra e sem julgamentos.

Assim como para os demais profissionais, o enfermeiro oncologista tem necessidade urgente de uma observação cuidadosa quanto às diversas práticas de medicina nãoconvencional. Anexá-las ao seu plano de cuidados sem uma análise criteriosa é temerário. Pode colocar em perigo a vida do paciente ao utilizar práticas para as quais ainda não se conhecem eficácia, segurança e possíveis interações com as terapias convencionais. Poderá ajudar o paciente ao oferecer informação precisa quanto à medicina não-convencional.

0 profissional de saúde não poderá fechar seus olhos frente a uma realidade que está se impondo: 0 uso das terapias não-convencionais. É mister uma reavaliação quanto à sua atitude, que deverá ser direcionada para um fortalecimento do seu papel de educador para a saúde.

\section{CONCLUSÕES}

- M ais de metade dos pacientes oncológicos do H ospital Universitário da Universidade de Brasília $(63,81 \%)$ usa medicina nãoconvencional. O s usuários dessa medicina são predominantemente mulheres, brancas, jovens, donas de casa, católicas, com o ensino fundamental incompleto e renda familiar em torno de seis salários mínimos. São residentes, em sua maioria, no D istrito Federal, dispondo de condições de moradia satisfatórias.

- A doença que predominou foi 0 câncer de mama, seguido dos linfomas.

- A fitoterapia foi a prática não-convencional mais utilizada.

- A indicação de terceiros foi o fator preponderante para o uso da medicina nãoconvencional.

- A maioria dos pacientes que fez uso da medicina não-convencional não abandonou 0 tratamento convencional.

- M ais de metade dos adeptos da medicina não-convencional $(55,23 \%)$ não informou à equipe médica que fazia uso desse tipo de tratamento.

- A quase totalidade dos entrevistados não recebeu quaisquer esclarecimentos em relação à medicina não-convencional.

- A maioria dos usuários de tratamentos nãoconvencionais $(89,55 \%)$ demonstrou desejo de receber orientação médica a respeito dessa medicina.

Assim, com base na experiência adquirida nesta pesquisa, consideramos importante enfatizar e fazer as seguintes recomendações: - H ánecessidade de uma melhor comunicação entre o profissional de saúde e o paciente. Os pacientes oncológicos carecem de uma informação adequada sobre sua doença e os diferentes tratamentos que são propostos.

- 0 paciente tem o direito de ser orientado quanto ao mecanismo de ação das diferentes modalidades terapêuticas não-convencionais, sua eficácia, indicações, contra-indicações, custo, procedência de produtos e efeitos adversos possíveis, assim como do risco de interações.

- 0 profissional de saúde deve ter uma atitude de neutralidade quanto ao uso da medicina não-convencional. Compreender a diversidade 
cultural, social e étnica do paciente pode ajudar a entender o que os pacientes procuram e esperam da medicina não-convencional. M elhor do que ignorar a prática de terapias não-convencionais é ter um conhecimento adequado das mesmas.

- 0 enfermeiro, antes de incluir os diferentes tratamentos não-convencionais no conjunto de assistência de enfermagem, precisa se instruir a respeito deles.

- Para a instrução dos alunos de ciências da saúde pode ser de grande auxílio uma disciplina em que se ministrem os fundamentos das terapias não-convencionais. - Recomenda-se o levantamento e a documentação, no prontuário médico, do uso de medicina não-convencional pelos pacientes oncológicos.

\section{REFERÊN CIAS BIBLIO G RÁFICAS}

1. Del Giglio A. Câncer: introdução ao seu estudo etratamento. São Paulo: Pasqualin; 1996.

2. Fletcher D M . U nconventional cancer treatments: professional, legal, and ethics issues. O ncol N ursForum 1992;19:1351-4.

3. von Gruenigen VE, H opkins M P. Alternative medicine in gynecologic oncology: a case re port. Gynecol 0 ncol 2000;77:190-2.

4. Corner J. Cancer nursing: a leading force for health care. J Adv N urs 1999;29(2):275-6.

5. Ernest $E, C$ assileth BR. H ow useful areunconventional cancer treatment? Eur J Cancer 1999;35:1608-13.

6. Cassileth BR, Chapman CC. Alternative and complementary cancer therapies. Cancer 1996;77(6):1026-34.

7. The role of alternative treatments for cancer. Johns H opkins M ed Lett $\mathrm{H}$ ealth After 50
2000;12:6-7.

8. Burstein H J. D iscussing complementary thera pies with cancer patients: what should we be talking about? J Clin O ncol 2000;18:2501-4.

9. Richardson MA, Sanders $T$, Palmer JL, Greisinger A, Singletary SE. Complementary/ alternative medicine use in a comprehensive cancer center and the implications for oncology. J Clin O ncol 2000;18:2505-14.

10. C assileth BR. Complementaries therapies: the american experience. Support $\mathrm{C}$ are $\mathrm{C}$ ancer 2000;8:16-23.

11. Söllner W, M aislinger S, D eVries A, Steixner E, Rumpold G, LukasP. Use of complementary and alternativemedicineby cancer patientsisnot associated with perceived distress or poor compliancewith standard treatment but with activecoping behavior. C ancer 2000;89:873-80.

12. M oore G. Woman and cancer: a gynecologic oncology nursing perspective. Available from: www.cancersource.com

13. Eisenberg D M , D avisRB, Ettner SL, Appel S, WilkeyS, Van Rompay M , Kessler RC. Trends in alternativemedicineusein the U nited States, 1990-1997. JAM A 1998;280:1569-75.

14. Bendazolli WS. Fitomedicamentos: perspectivas de resgate de uma terapia histórica. M undo Saúde 2000;24:123-6.

15. Brandäo M GL, FreireN, V ianna-Soares CD. Vigilância de fitoterápicos em M inas G erais: verificaçäo da qualidade dediferentes amostras comerciais de camomila. Cad Saúde Pública 1998;14:613-6.

16. Batistic M A, Auricchio MT, Hoppen VR, Yamashita IY. Verificaçäo da qualidade e identidade dechásmedicinais. Rev Inst Adolfo Lutz 1989;49:45-9.

17. H outsPS, H ermann JF. Educational needs related to complementary and alternative therapies. C ancer Pract 1999;7:262-5. 